\title{
Análisis y evaluación de marcos de trabajo para el desarrollo de aplicaciones y servicios en la nube PAAS
}

\author{
Rafael Fernández Domínguez ${ }^{1, *}$, Giner Alor Hernández ${ }^{1}$, Leticia Dávila Nicanor ${ }^{2}$ \\ ${ }^{1}$ División de Estudios de Posgrado e Investigación Instituto Tecnológico de Orizaba, Veracruz, \\ México. \\ rafafallol@gmail.com, galor@itorizaba.edu.mx \\ ${ }^{2}$ Maestría en ciencias de la computación Centro Universitario UAEM Valle de México, Atizapan de \\ Zaragoza, Edo de México, México. \\ letidn@yahoo.com.mx
}

\begin{abstract}
Resumen. Hoy en día, las tecnologías para la Internet aumentan a pasos agigantados, provocando la existencia de un gran número de proveedores de servicios en la nube como: Google App Engine, Windows Azure, Amazon E2C, entre otros. Esto a su vez hace que las empresas adoptantes sean más exigentes al adquirir un servicio. En este sentido, este trabajo propone un análisis comparativo entre los diferentes marcos de trabajo para el desarrollo de servicios y aplicaciones en la nube, considerando aspectos de calidad de servicio (QoS) como: Accesibilidad, Confiabilidad, Escalabilidad, Elasticidad, Disponibilidad, Adaptabilidad, Portabilidad y Tiempo de Respuesta, que permita a los clientes seleccionar un marco de trabajo para el desarrollo de aplicaciones de acuerdo a sus necesidades.
\end{abstract}

Palabras Clave: Cloud Computing, QoS, PaaS, Frameworks.

\section{$1 \quad$ Introducción}

La computación en la nube (Cloud Computing) se define como un modelo que permite el acceso de red conveniente y bajo demanda, de un conjunto compartido de recursos informáticos configurables (por ejemplo, redes, servidores, almacenamiento, aplicaciones y servicios) que son rápidamente aprovisionados y liberados con un mínimo esfuerzo de gestión o interacción de servicios [1,2]. Es una tecnología orientada a la Web, y surge para combinar ciertos aspectos para la mejora en el almacena-miento y ventajas de las empresas, como la reducción de costos, de producción y de mantenimiento. Se confunde fácilmente con el Grid Computing que surge a mediados de 1990, se deriva de la red de energía eléctrica para destacar sus características como la capacidad de penetración, sencillez y fiabilidad. La demanda a gran escala de aplicaciones científicas requieren más po- 
der de cómputo que un grupo dentro de un mismo dominio (por ejemplo, un instituto) podría proporcionar; y debido a la inter-conexión rápida por Internet, institutos científicos fueron capaces de compartir y agregar recursos geográficamente distribuidos, incluidos los sistemas de racimo, las instalaciones de almacenamiento de datos y fuentes de datos pertenecientes a diferentes organizaciones [3]. Grandes empresas de Software forman parte de esta tecnología brindando diversos servicios a los usuarios, estos servicios se clasifican en tres tipos diferentes: PAAS, SAAS e IAAS [1, 2].

En este caso la Plataforma como servicio (PaaS) ofrece un entorno de desarrollo como un servicio, donde las aplicaciones se desarrollan utilizando un conjunto de lenguajes de programación y herramientas. Estos servicios incluyen almacenamiento de desarrollo, integración, pruebas o recursos para completar el ciclo de vida de servicios [1]. El consumidor no gestiona ni controla la infraestructura de nube subyacente, incluyendo la red, servidores, sistemas operativos, o el almacenamiento, pero tiene el control sobre las aplicaciones implementadas y posiblemente de alojamiento de aplicaciones configuraciones de entorno [2];

Este análisis comparativo tiene la finalidad de dar soporte a los nuevos clientes del cómputo en la nube al elegir un marco de trabajo para el desarrollo de aplicaciones, al presentar una comparación entre 5 marcos de trabajo bajo el soporte de atributos de calidad de servicio. La calidad del servicio (QoS), la cual es definida por el ITU Telecomunication Standardization Sector (ITU-T) como "El efecto colectivo de la eficiencia de servicios que determinan el grado de satisfacción de un usuario del mismo servicio." [4].

\section{Trabajos relacionados}

En años recientes se han propuesto diversos trabajos enfocados a la computación en la nube, a continuación se presentan algunos de ellos. En [5] se presenta un estudio de diversos servicios en la nube. Se identifican y ex-plican sus principales características, posteriormente se organizan estas características y se propone una taxonomía con estructura de árbol basándose en taxonomías y en tablas de comparación existentes. En [6] se plantea el desarrollo de un marco de trabajo llamado (SMI) Service Measure Index, que consiste en la aplicación de medidas coherentes y significativas que se diseñaron para permitir la comparación de los actuales servicios basados en la nube o proveedores de servicios disponibles. En [7] se presenta de manera general el concepto, la historia, las ventajas y desventajas de la computación en la nube, así como el esfuerzo para crear una normalización de la misma. En [8] se examinan algunas perspectivas de Computación en la nube así como algunas características esenciales de la misma, basado en definiciones dadas por el NIST (National Institute of Standard and Tecnology) c En [9] se propone un marco de trabajo y un mecanismo que mida la calidad y dé prioridad a servicios en la nube, ya que debido a la gran diversidad de servicios en la nube disponibles es difícil decidir qué servicio utilizar y en qué se basa para su selección, puesto que hoy en día no existe algún marco de trabajo que permita evaluar dichos servicios. En [10] se propone una arquitectura para la asignación de recursos orientada a la alta calidad del servicio y el cumplimiento 
del Service Level Agreements (SLA).En [11] se proporciona información a detalle de criterios de seguridad que los próximos clientes de los servicios en la nube considerarán para seleccionar de manera eficiente una nube pública o un Cloud Service Provider (CSP) que satisfaga sus necesidades, En [12] se plantea el objetivo de dar una mejor comprensión del tema identificando y clasificando las principales preocupaciones y soluciones de seguridad en la nube, de igual manera, se propone una taxonomía dando una visión general de lo que es la seguridad actualmente en la computación en la nube. En [13] se revisan los desafíos relacionados con los conceptos de confianza y gestión de Service Level Agreements (SLA) y se analizan los marcos existentes de los acuerdos de nivel de servicio en diferentes ámbitos, como los servicios Web y la red En [14] se propone un marco de trabajo de comparación que contiene las características de Computación en la nube. Este marco de trabajo se aplica en cuatro modelos de procesos establecidos para la ingeniería de requerimientos que son RUP, XP, Volere y VModel, con el fin de estudiar a qué grado se aplican a los requisitos específicos de las soluciones basadas en la nube.

\section{Atributos de calidad aplicables a servicios en la Nube}

Los atributos de calidad son aquellos puntos que brindan a los usuarios la confianza al seleccionar un marco de trabajo para el desarrollo en la nube. La universidad Carnegie Mellon en Silicon Valley California dio comienzo a un consorcio que definiera un marco de trabajo que señale atributos y métricas para la evaluación de calidad para seleccionar el servicio y el proveedor más conveniente. Este consorcio lleva por nombre "Cloud Service Measurement Initiative Consortium" (CSMIC). Está conformado por un conjunto de instituciones de todo el mundo que abogan por mejorar el cómputo en la nube. Este consorcio desarrolló el "Service Measurement Index" (SMI), que es un marco de trabajo que proporciona un índice de medición de los servicios en la nube basado en métricas orientada a la calidad del servicio [15]. Al identificar los atributos de calidad, se llevó a cabo la selección de los más relevantes con base en el soporte que proporciona la literatura actual al respecto de ellas (artículos científicos y libros) y a continuación se describen:

-Adaptabilidad: La capacidad del proveedor de servicios para adaptarse a los cambios en las necesidades del cliente.

- Disponibilidad: La adecuación de la ventana de la disponibilidad del servicio, así como la probabilidad de que en realidad será siempre la ventana de disponibilidad a los clientes.

—Elasticidad: La capacidad de un proveedor de servicio en la nube, para ajustar el consumo de recursos para un servicio a una velocidad suficientemente rápida para satisfacer la demanda del cliente.

- Tiempo de respuesta del Servicio: Un indicador del tiempo entre cuando se solicita un servicio, y cuando la respuesta está disponible. 
- Portabilidad: La capacidad de un cliente para mover fácilmente un servicio de un proveedor de servicio en la nube a otro.

- Escalabilidad: La capacidad de un proveedor de servicios cloud para aumentar o disminuir la cantidad de servicios disponibles.

-Accesibilidad: El grado en que un servicio es operado por los usuarios

- Confiabilidad: Refleja la medida de cómo un servicio funciona sin falla bajo condiciones dadas durante un periodo de tiempo determinado[15]

Después de analizar 40 diferentes propuestas de solución de varios autores para llevar a cabo la evaluación de los marcos de trabajo basados en métricas de calidad, se propusieron las siguientes formas de evaluación para realizar el análisis, las cuales se muestran en la tabla 1

Table 1. Formas de evaluación propuestas para la evaluación de las métricas de calidad seleccionadas.

\begin{tabular}{|c|c|}
\hline Atributo & Propuesta de evaluación (métrica) \\
\hline \multicolumn{2}{|l|}{ Accesibilidad } \\
\hline & $A c=F$ \\
\hline & $\begin{array}{l}\text { Donde F representa la facilidad que poseen los proveedores de } \\
\text { servicios en la nube para que nuevos usuarios se adapten a ellos. }\end{array}$ \\
\hline \multicolumn{2}{|l|}{ Portabilidad } \\
\hline & $P=m$ \\
\hline & $\begin{array}{l}\text { En la cual se representa la cantidad de marcos de trabajo (m) que } \\
\text { soportan aplicaciones desarrolladas en otros marcos }\end{array}$ \\
\hline \multicolumn{2}{|l|}{ Escalabilidad } \\
\hline & $\frac{\sum_{t=2}^{n} c_{1}}{n}$ \\
\hline & $\begin{array}{l}\text { Donde c representa los resultados en milisegundos obtenidos en la } \\
\text { evaluación sobre n que representa la cantidad de campos utilizados } \\
\text { para la sumatoria }\end{array}$ \\
\hline \multicolumn{2}{|l|}{ Adaptabilidad } \\
\hline & $A=T P I-T P A$ \\
\hline & $\begin{array}{l}\text { Diferencia de tiempo promedio al adaptar la aplicación a nuevos } \\
\text { cambios, donde: TPI=Tiempo promedio inicial. }\end{array}$ \\
\hline \multicolumn{2}{|r|}{ (1) } \\
\hline & $\frac{\sum_{t=2}^{n} c_{1}}{n}$ \\
\hline & $\begin{array}{l}\text { Se calcula la sumatoria de los resultados obtenidos en milisegun- } \\
\text { dos(c) desde i que es el valor inicial hasta n que representa el total }\end{array}$ \\
\hline
\end{tabular}




\begin{tabular}{|c|c|}
\hline Atributo & Propuesta de evaluación (métrica) \\
\hline & de campos utilizados en cada. \\
\hline \multicolumn{2}{|c|}{ Disponibilidad } \\
\hline & $\frac{\sum c}{n}$ \\
\hline & $\begin{array}{l}\text { Donde se refleja el tiempo promedio al realizar la sumatoria de } c \\
\text { que representa los milisegundos obtenidos como resultado sobre la } \\
\text { cantidad de campos utilizados para la sumatoria } \mathrm{n} \text {. }\end{array}$ \\
\hline \multicolumn{2}{|c|}{ Tiempo de Respuesta } \\
\hline & EiTi/n \\
\hline & $\begin{array}{l}\text { Con esta fórmula se obtiene el tiempo promedio de respuesta al } \\
\text { realizar la sumatoria de los tiempos pintados como resultado (Ti) } \\
\text { sobre la cantidad de campos utilizados (n) en la sumatoria. }\end{array}$ \\
\hline \multicolumn{2}{|c|}{ Confiabilidad } \\
\hline & $C=\mathrm{cm}$ \\
\hline & $\begin{array}{l}\text { Donde } \mathrm{cm} \text { es la cantidad máxima de interacciones soportadas en } \\
\text { los diferentes marcos de trabajo. }\end{array}$ \\
\hline
\end{tabular}

\section{$4 \quad$ Marcos de trabajo para el desarrollo de servicios y aplicaciones para la computación en la nube PAAS}

Los marcos de trabajo orientados al desarrollo de aplicaciones para la nube permiten la creación de aplicaciones capaces de desplegarse en las mismas. Existe gran variedad de marcos de trabajo, a continuación se presentan los seleccionados para este trabajo con una breve descripción:

Google App Engine: Es una oferta PaaS, que proporciona soporte para Python y Java. AppEngine permite crear aplicaciones Web escalables, sin necesidad de hardware complejo subyacente y capas de software. Google abstrae esas capas y permite concentrarse plenamente en la aplicación [16].

Microsoft Azure: La plataforma de servicios Azure es la oferta PaaS de Microsoft. Azure tiene una API específica para almacenar y recuperar datos llamados Services de SQL. El sistema subyacente para estos servicios SQL es Microsoft SQL Server. Aunque no todas las funcionalidades de Microsoft SQL Server están expuestas a través de la API, el usuario ejecuta las operaciones y el uso de un lenguaje de con-sulta SQL restringido [17].

Heroku: Es una muy poderosa plataforma basada en Web que proporciona soporte para Ruby, JavaScript y Java Web. Se despliega directamente en un entorno PaaS, permite la migración rápida desde el desarrollo a la etapa de implementación. También contiene un editor de colaboración en tiempo real para su uso con un máximo de cinco personas [18]. 
Amazon Elastic MapReduce: Es un servicio Web de Amazon (AWS), que utiliza Hadoop para ofrecer una funcionalidad MapReduce. En el fondo sí se basa princi-palmente en otros dos servicios AWS: Elastic Compute Cloud (EC2) y Simple Sto-rage Service (S3) [19].

CloudFoundry: Es una plataforma de código abierto como un servicio. Entorno ofrecido por VMware, proporciona el entorno para albergar múltiples lenguajes y marcos en una pila abierta de aplicaciones de software que se ejecutan tanto fuera como dentro del firewall. Las principales características de Cloud Foundry son: Elección de los marcos de desarrollo, elección de servicios de infraestructura de aplicaciones, y la elección de las nubes [20].

\section{$5 \quad$ Análisis comparativo}

En esta sección se describen los diferentes casos de estudio propuestos para llevar a cabo el desarrollo de las evaluaciones de la calidad de servicio de los marcos de trabajo en cada uno de los atributos de calidad seleccionados para este trabajo, de la misma manera se presentan unos ejemplos de gráficas de resultados obtenidos en las evaluaciones desarrolladas.

Accesibilidad.- En el caso de la accesibilidad, se desarrolló una aplicación que tiene lugar siempre al comienzo en la vida del desarrollador de software, un simple y sencillo "Hola mundo" en cada plataforma soportada por cada marco de trabajo; para llevar a cabo la evaluación se consideró una escala de Likert de 3 valores con los siguientes elementos: (1) "Alto", el cual significa que cuenta con sencillez en el proceso de navegación, fácil acceso a las herramientas para desarrollo, depuración y despliegue, documentación abundante para comprender y aprender cómo funciona dicho marco; (2) "Medio" que es el nivel que contempla una navegación sencilla, se obtienen las herramientas proporcionadas fácilmente pero no cuenta con la documentación apta para principiantes; (3) "Bajo". En este nivel se encuentran los marcos de trabajo más complejos ya que su navegación es agotadora y las herramientas que proporciona son demasiado complejas para utilizarlas si no se tiene capacitación previa.

Portabilidad.- En el caso de la portabilidad, se desarrolló una aplicación en los diferentes marcos de trabajo, se empaquetaron como proyectos y se dispuso a transferir las aplicaciones de un marco de trabajo a otro y, de esa forma, evaluar el soporte de cada marco de trabajo para aceptar aplicaciones desarrolladas en otros ambientes de desarrollo de aplicaciones.

Tiempo de respuesta. Al llevar a cabo la evaluación del tiempo de respuesta de los marcos de trabajo para el desarrollo de aplicaciones se desarrolló una aplicación Web en cada marco de trabajo, en la cual se realizó un proceso de conexión a la base de datos correspondiente a cada marco de trabajo y realizar la inserción, modificación y elimina- 
ción de un registro simultáneamente y obtener así los tiempos de respuesta del proceso de cada iteración a la base de datos.

Disponibilidad.- Al llevar a cabo la evaluación de la Disponibilidad, se desarrolló una aplicación Web en cada marco de trabajo en la cual se consume el WS basado en SOAP, el cual devuelve una instancia con los datos climatológicos según la ciudad que el usuario solicite. Mediante un formulario, se envía una clave ZIP de la ciudad en EE.UU. de la que el usuario requiera conocer la información climatológica y la recepción de la información que se muestra en la pantalla del navegador. Esta aplicación al mismo tiempo de solicitar y recibir la información, también calcula el tiempo del proceso de petición y de respuesta del WS.

Escalabilidad.- Al desarrollar una aplicación Web en cada uno de los marcos de trabajo seleccionados. La escalabilidad consta de incrementar la funcionalidad de la aplicación, es decir, hacer que uno de sus componentes crezca funcionalmente en carga. La evaluación consiste en consumir 5 servicios Web SOAP diferentes, comenzando con uno e ir incrementando los servicios consumidos hasta llegar a 5, esto se realiza con la opción de un control "select" en HTML, donde el usuario elige con cuantos WS quiere calcular el tiempo de respuesta. En la figura 2 se presentan los resultados obtenidos en la evaluación realizada a los marcos de trabajo para obtener los niveles de escalabilidad reflejados por cada uno en la siguiente grafica donde se observa la potencia que ofrece Azure al presentar mejores tiempos de respuesta.

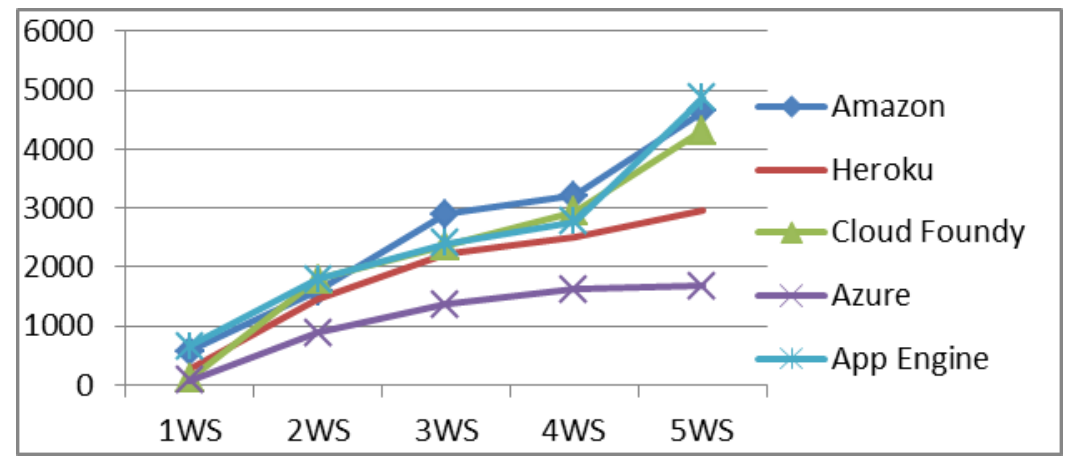

Fig. 1. Resultado de escalabilidad en los marcos de trabajo

Elasticidad.- Se desarrolló una aplicación Web en cada marco de trabajo que incremente la capacidad de memoria al concretizar una clase un número determinado de veces, en la cual se consumen 2 servicios Web SOAP y son almacenados los datos en una base de datos calculando el tiempo demorado para que se concluya el proceso. 
En la figura 2 se observan los resultados obtenidos al evaluar la elasticidad en los marcos de trabajo reflejando a Azure como la mejor opción.

Confiabilidad.- Para este caso de estudio, se desarrolló una aplicación Web que consuma un WS tantas veces como sea soportado por el marco de trabajo hasta que se obtenga un fallo por parte del mismo mediante un proceso simple, en el cual se inserta la cantidad de iteraciones requeridas por el usuario en una caja de texto, al dar clic en el botón "calcular" realiza un ciclo con la cantidad de iteraciones solicitadas y en cada una busca concretizar una clase.

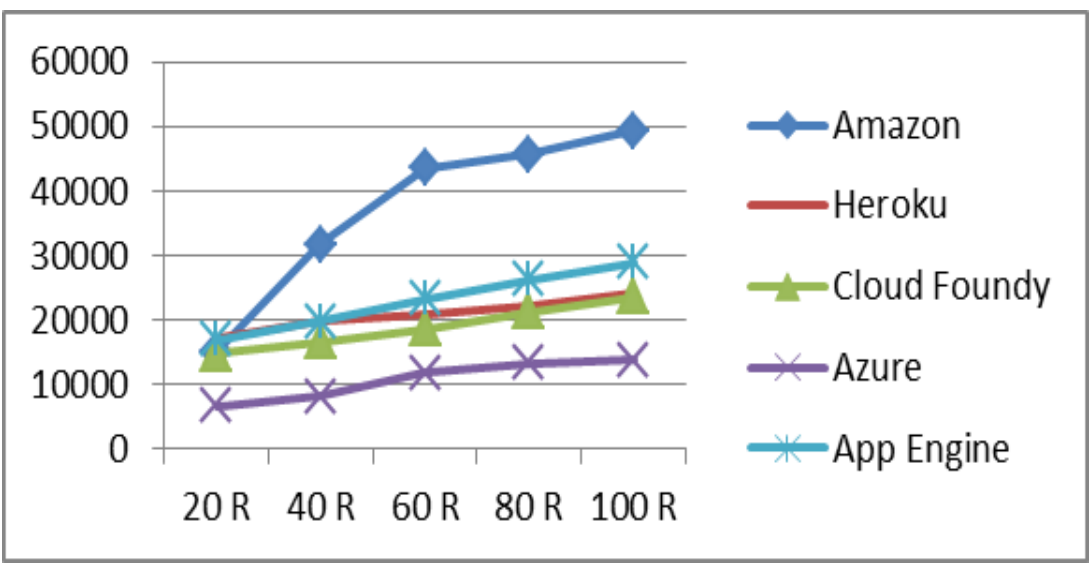

Fig. 2. Resultado de la Elasticidad en los marcos de trabajo

En la tabla 2 se muestran los resultados de las pruebas realizadas sobre los marcos de trabajo, en la cual se presentan las métricas de calidad seleccionadas para este trabajo de tesis y los marcos de trabajo seleccionados para ser evaluados.

Cada sección se encuentra dividida por métrica de calidad en la parte superior de la tabla, las cuales se subdividen en 3 niveles: "BUENO, MEDIO, MALO". Estos niveles definen como:

- "Bueno": como el marco de trabajo que obtuvo un resultado sobresaliente a los demás alcanzando niveles más altos en las evaluaciones desarrolladas.

- "Medio": como el marco de trabajo que obtuvo un nivel medio en los resultados obtenidos durante las evaluaciones. 
Análisis y evaluación de marcos de trabajo para el desarrollo de aplicaciones y servicios ...

— "Malo": implica que obtuvieron el resultado más bajo durante la evaluación. Estos niveles permiten conocer qué marcos de trabajo mantienen un nivel más apto para los usuarios que desean iniciar el uso de los mismos.

Table 2. Matriz de resultados

\begin{tabular}{|c|c|c|c|c|c|c|}
\hline GAE & CF & AZURE & AMAZON & HEROKU & & \\
\hline \multirow{3}{*}{$x$} & \multirow{3}{*}{$x$} & $x$ & $x$ & $x$ & BUENO & \multirow{3}{*}{ Accesibilidad } \\
\hline & & & & & MEDIO & \\
\hline & & & & & MALO & \\
\hline \multirow{5}{*}{$x$} & \multirow{5}{*}{$x$} & $x$ & & $x$ & BUENO & \multirow{3}{*}{ Portabilidad } \\
\hline & & & & & MEDIO & \\
\hline & & & $x$ & & MALO & \\
\hline & & $x$ & & & BUENO & \multirow{3}{*}{ Disponibilidad } \\
\hline & & & & $x$ & MEDIO & \\
\hline \multirow[t]{3}{*}{$x$} & & & & & MALO & \\
\hline & $x$ & & & & BUENO & \multirow{3}{*}{ Adaptabilidad } \\
\hline & & $x$ & & & MEDIO & \\
\hline \multirow[t]{9}{*}{$x$} & & & & & MALO & \\
\hline & \multirow{3}{*}{$x$} & $x$ & & & BUENO & \multirow{3}{*}{ Confiabilidad } \\
\hline & & & & & MEDIO & \\
\hline & & & & $x$ & MALO & \\
\hline & \multirow{3}{*}{$x$} & & & $x$ & BUENO & \multirow{3}{*}{$\begin{array}{l}\text { Tiempo de } \\
\text { Respuesta }\end{array}$} \\
\hline & & & & & MEDIO & \\
\hline & & $x$ & & & MALO & \\
\hline & & $x$ & & & BUENO & \multirow{3}{*}{ Escalabilidad } \\
\hline & $x$ & & & & MEDIO & \\
\hline \multirow[t]{4}{*}{$x$} & & & & & MALO & \\
\hline & & $x$ & & & BUENO & \multirow{3}{*}{ Elasticidad } \\
\hline & & & & $x$ & MEDIO & \\
\hline & & & $x$ & & MALO & \\
\hline
\end{tabular}




\section{Trabajo a Futuro y Conclusiones}

Los marcos de trabajo para el desarrollo de aplicaciones y servicios en la nube enfrentan la problemática de la poca existencia de información respecto a la calidad del servicio en el cómputo en la nube, además de que no hay un precedente de la existencia de material que reúna información de diversos marcos de trabajo o que comparen el soporte ofrecido por cada uno con base en métricas de calidad especializadas, siendo una buena aportación el presente proyecto. Llegando a la conclusión de que falta mucho por hacer en el ámbito del cómputo en la nube y se recomienda analizar otros criterios de calidad semejantes a los analizados en el presente trabajo como capacidad, flexibilidad, funcionabilidad, entre otros, y con ello aportar productos académicos que apoyen la estandarización de la calidad del servicio en la nube.

\section{Referencias}

1. María del Pilar Salas, Luis Omar Colombo, Cloud Computing: A Review of PaaS, IaaS, SaaS Services and Providers. Lámpsakos No. 7, Medellin, Colombia, pp. 47-57 (2012)

2. Mark Lee Badger, Timothy Grance, Robert Patt-Corner, Jeffery M, Voas. Cloud Computing Synopsis and Recommendations, Technical Report. NIST, Gaithersburg, MD, United States, pp 0-81 (2012)

3. Christof Weinhardt, Arun Anandasivam, Benjamin Blau, et al., Cloud Computing - A Classification, Business Models, and Research Directions, Business \& Information Systems Engineering volumen 5, Karlsruhe, Germany. pp 392-399 (2009)

4. Jan Øyvind Aagedal, Quality of Service Support in Development of Distributed Systems, Faculty of Mathematics and Natural Sciences, University of Oslo, March 9, (2001)

5. C.N. Höfer, G. Karagiannis, Cloud computing services: taxonomy and comparison, In processing of the GLOBECOM Workshops (GC Wkshps), IEEE, Springer. Enschede, the Netherlands. pp 81-94. (2010)

6. Jane Sigel, Jeff Perdue, Cloud Services Measures For Global Use, The Service Measure Index (SMI), In Proceedings of the Service Research and Innovation Institute Global Conference, IEEE. Mountain View, CA, USA, pp. 411-415, (2012)

7. Ling Qian, Zhiguo Luo, Yujian Du, Leitao Guo, Cloud Computing: An Overview, in procceding of the Cloud Computing, First International Conference, CloudCom, Springer-Verlag Berlin Heidelberg. Beijing, China. pp. 626-631, (2009)

8. Vic (J.R.) Winkler, Chapter 2- Cloud Computing Architecture, Securing the Cloud, Cloud Computer Security Techniques and Tactics, Elsevier. Waltham, Massachusetts, USA, pp 29$53,(2011)$

9. Saurabh Kumar, Steve Versteeg, Rajkumar Buyya. A framework for ranking of cloud computing services. Future Generation Computer Systems, Volume 29, Elsevier. Melbourne, Australia. Pp 1012-1023, (2012) 
10. Kouessi Arafat Sagbo, Pélagie Houngue. Quality architecture for resource allocation in cloud computing. In Proceedings of the First European conference on Service-Oriented and Cloud Computing (ESOCC'12), Flavio Paoli, Ernesto Pimentel, and Gianluigi, (2012)

11. Vic (J.R.) Winkler. Chapter 8 - Security Criteria: Selecting an External Cloud Provider. Securing the Cloud, Cloud Computer Security Techniques and Tactics, Elsevier. Waltham, Massachusetts, USA, pp 211-232, (2011)

12. Nelson Gonzalez, Charles Miers, Fernando Redigolo, Marcos Simplicio, Tereza Carvalho, Mats Naslund, Makan Pourzandi. A quantitative analysis of current security concerns and solutions for cloud computing, in Proceedings of the IEEE Third International Conference on Cloud Computing Technology and Science CLOUDCOM '11. Washington, DC, USA, pp 231-238, (2011)

13. Mohammed Alhamad, Tharam Dillon,, Elizabeth Chang, A Survey on SLA and Performance Measurement in Cloud Computing. In processing of the On the Move to Meaningful Internet System (OTM2011). Springer, Australia, pp. 469-477, (2011)

14. Stefan Wind, Schrödl, Requirements Engineering for Cloud Computing: A Comparison Framework. In Proceedings of The Workshop on Information Systems Economics (WISE2010), Springer-Verlag Berlin Heidelberg. Augsburg, Germany, pp. 404-415, (2010)

15. Jane Sigel, Jeff Perdue, A Cloud Services Measures For Global Use, The Service Measure Index (SMI). In Proceedings of the Service Research and Innovation Institute Global Conference, IEEE. Mountain View, CA, USA, pp. 411-415, (2012)

16. Google team. 26-mar-2012. Google App Engine, 23-Feb-2013, https://developers. google.com /appengine/?hl=es, (2012)

17. Microsoft team. 23-Feb-2013, Windows Azure: Plataforma en la nube de Microsoft., 23-Feb2013, http://www.windowsazure.com/es-es/

18. Heroku team. 23-Feb-2013, Heroku Cloud Application Platform, 23-Feb-2013, http://www .heroku.com/, (2013)

19. Amazon Web Services team, Amazon Elastic MapReduce (Amazon EMR), 23-Feb-2013 http://aws.amazon.com/elasticmapreduce/, (2011)

20. VMware team. 24-Ene-2013, Cloud Foundry, 23-Feb-2013, http://www.cloudfoundry .com/ , (2013) 